\title{
Prediction of battery depletion in implanted pacemakers
}

\author{
GEOFFREY DAVIES and HAROLD SIDDONS
}

\author{
St. George's Hospital, London S.W.1
}

By the use of a measuring oscilloscope and the standard electrocardiogram limb leads the degree of battery depletion in an implanted pacemaker can be estimated. A formula based on readings obtained by this means has been used to determine when Devices fixed rate pacemakers should be removed. Laboratory tests show that $90 \%$ of their useful life is obtained by this means and it proved possible to extend the period of implantation from an arbitrary 24 months to 25 to 34 months without failure from battery depletion.

With available batteries as the power source for implanted pacemakers, battery depletion is inevitable within a few years. The consequent cessation of pacing may result in prolonged asystole and, it cannot be doubted, is responsible for some sudden deaths. This length of useful life of a pacemaker has been found to vary from patient to patient so that if all pacemakers were removed after an arbitrary period estimated to precede the first failures from depletion a considerable period of useful life would be wasted. This implies that operations for change of pacemaker would have to be carried out at more frequent intervals than would be necessary if battery depletion could be predicted in each individual patient. The problem is to devise a method by which the degree of battery depletion can be determined in an implanted pacemaker.

\section{PRESENT METHODS}

Many methods have been described. Radiographs of the implanted unit (Chardack et al., 1964) have not proved accurate enough. Greater success has been achieved by designing the circuitry of pacemakers so that there is a significant slowing when battery depletion is impending. Recent models made by some manufacturers have circuitry designed so that very small changes of rate become significant. These changes are of a degree which require electrical measurement. Several North American and other clinics now run telephone services by which the hospital can record electrically the pacemaker rate, the patient having applied over his pacemaker a special device which he attaches to his telephone. It is not yet known how efficient this method is in terms of $(a)$ how much useful life is left in the pacemaker when it is removed on these criteria, and $(b)$ how often pacing ceases before the removal. In Devices pacemakers a change of rate of $5 \%$, which is of a degree measurable by the patient or doctor, should be regarded as an indication of a fault in the pacemaker and the need for its removal, but lesser changes in rate are not likely to be significant. In these units an alteration of pulse width is indicative of battery depletion but electronic facilities are required to measure this.

\section{PROPOSED METHOD}

We have approached the problem of predicting battery depletion by detailed examination of the impulse curve displayed on a measuring oscilloscope (Davies and Siddons, 1969). Before applying our experience with this technique it was necessary to build up data on which to base the $\frac{D}{0}$ prediction in specific pacemaker models. Therefore, when the Devices TF 2970 model was first N introduced at St. George's Hospital, all were taken out after an arbitrary period of 24 months. On O removal they were placed in a saline bath at $\mathrm{\omega}$ $37^{\circ} \mathrm{C}$ with an electrode to simulate continued use. Observation revealed that battery exhaustion occurred at between 29 and 42 months, suggesting $\mathbb{D}$ that many of the units on removal still had a ? number of months of useful life.

The serial stimulus curve measurements which had been recorded in all patients up to the arbitrary 24 months were correlated with the full $\stackrel{\unrhd}{\Omega}$ life as determined in the laboratory. A formula which could be applied to subsequent patients 
with implants was devised from the following considerations :

The design of Devices 2970 pacemakers is such that provided the electrical impedance between the electrodes exceeds a certain value, the unit has a fixed output voltage. However, should this impedance fall, the pacemaker maintains a fixed current output (with a corresponding drop in output voltage) and it is for this reason that the unit is usually referred to as having current limiting characteristics. The wave form obtained on the measuring oscilloscope using a pacemaker with current limiting characteristics shows (Fig. 1) either (a) the unit is working in a current limit-

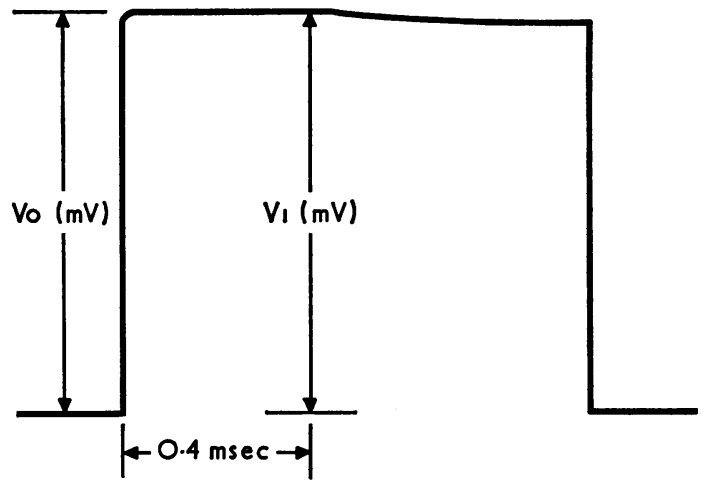

(a)

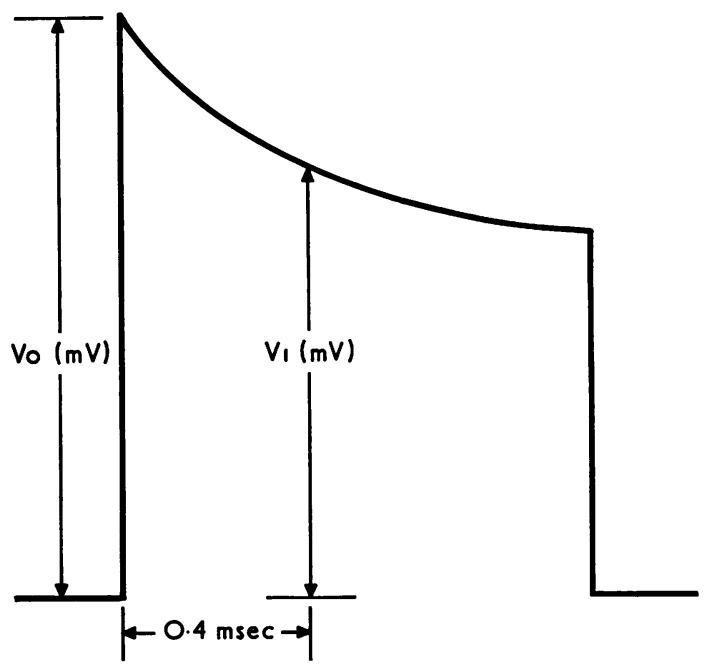

(b)

FIG. 1. The electrical impulse from an implanted pacemaker as recorded on an oscilloscope using an ECG standard configuration: (a) current limiting of the pacemaker, and (b) non-current limiting. ing mode and hence maximum current is being used or $(b)$ the impedance of the system is such that current limiting is not taking place. The decay ratio $V_{1} / V_{0}$ bears a relationship to the current being used in the system and so provides a means of indirectly measuring this factor. The current to which a particular unit is limited is given in the manufacturer's test report. As the pacemaker will be using power from the batteries irrespective of whether it is implanted or not, a normalizing factor has to be applied to the decay ratio which is obtained from Figure 2 . The graph is drawn in a way which attempts to compensate for the efficiency of pacemakers with different current limiting parameters.

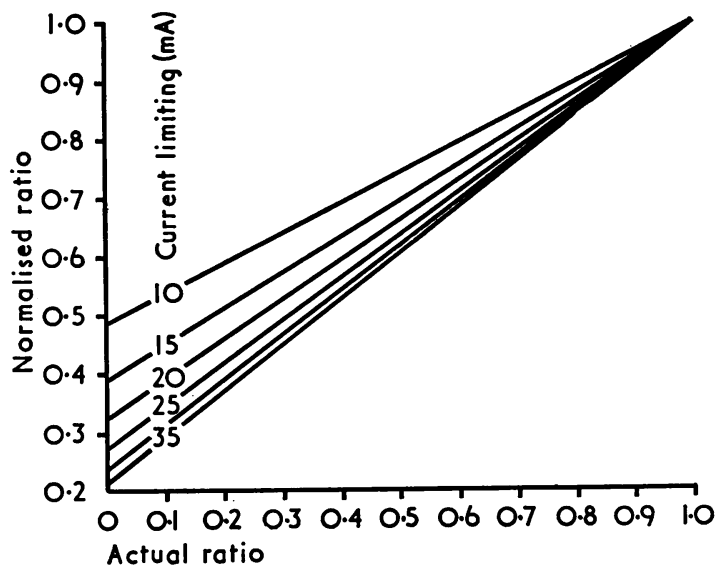

FIG. 2. A graph enabling the recorded decay ratio (actual ratio) to be converted to a normalized ratio for pacemakers with different amounts of current limiting. Example: actual ratio $0 \cdot 75$, current limiting $15 \mathrm{~mA}$, the normalized ratio from the graph is 0.85 . If the current limiting is $35 \mathrm{~mA}$, the normalized ratio becomes 0.80 .

Taking the product of time in use in weeks $(t)$, the pulse width in milliseconds $(w)$, the pulse rate per minute $(r)$, the current limiting in milliamperes $(c)$, and the normalized decay ratio $(s)$ gives a term which has been called the ' $D$ ' factor. It has been found empirically that with Devices TF 2970 units battery exhaustion occurs when this figure reaches $125,000 \pm 10 \%$.

In the case of a pacemaker which has been implanted for 106 weeks with a rate of $68 /$ minute, a pulse width of $0.98 \mathrm{msec}$, a normalized decay ratio of 0.87 , and current limiting of $13 \mathrm{~mA}$, the ' $D$ ' factor $t \times r \times w \times s \times c$ is : $106 \times 68 \times 0.98 \times 0.87$ $\times 13=79,892$. By proportion the total expected useful life of the unit may then be calculated as 
$\frac{106 \times 125,000}{79,892}$ or 166 weeks ( 38 months). Alternatively, the following formula may be used:

expected pacemaker life (in weeks) $=\frac{125,000}{\text { rate } \times \text { pulse width } \times \text { normalized decay } \times \text { current limiting }}$

\section{CURRENT ROUTINE}

During the first two years patients are being seen at intervals of 4 to 10 months or when the patient or doctor has noted a change in stimulus rate of $5 \%$. After the two-year period we see patients at decreasing intervals according to the $\mathrm{D}$ factor calculation, two or three visits usually being required before we decide on a change of pacemaker. It has proved of value to make an oscilloscopic recording at all visits. Not only do more recordings provide firmer data on which to base the prediction of battery depletion but they may provide other important information.

\section{RESULTS FROM METHOD}

So far this formula has been applied prospectively in 18 patients with fixed-rate pacemakers. It has been possible with confidence to leave pacemakers in for from 25 to 34 months and none has stopped pacing from battery exhaustion. This gain, probably utilizing $90 \%$ of the available power, represents an increased implanted life of $17 \%$ over our previous practice of removal at 24 months.

\section{CONCLUSIONS}

In order to obtain as much life out of a pacemaker as possible it is necessary to predict battery deple- tion for each individual patient. An oscilloscopic technique has been evolved by which it is possible to predict battery depletion before the natural ageing of the battery drops the output below the level for pacing.

Working with fixed-rate Devices 2970 pace- i makers, a formula has been deduced from collected data by which the application of $90 \%$ of battery life is used before the pacemaker is changed.

Prediction of battery life in Demand pacemakers is inevitably more difficult than in fixed-rate models as the current drain must depend on the proportion of time for which it is suppressed and therefore not putting out stimuli. This additional factor is in itself unpredictable and renders the method of prediction here described less accurate, but we believe that it will be of some value.

We should like to thank the Board of Governors of St. George's Hospital for the aid provided for this research project.

\section{REFERENCES}

Chardack, W. M., Gage, A. A., Frederico, A. J., Schimert, G., and Greatbatch, W. (1964). Clinical experience with an implantable pacemaker. Ann. N.Y. Acad. Sci., 111, 1075.

Davies, J. G., and Siddons, H. (1969). The detection of impending failure in implanted pacemakers. Thorax, 24, 74 